\title{
Numerical Solution of Partial Integrodifferential Equations of Diffusion Type
}

\author{
Imran Aziz and Imran Khan \\ Department of Mathematics, University of Peshawar, Peshawar, Pakistan \\ Correspondence should be addressed to Imran Aziz; imran_aziz@upesh.edu.pk
}

Received 30 June 2017; Accepted 24 October 2017; Published 15 November 2017

Academic Editor: Kishin Sadarangani

Copyright ( 2017 Imran Aziz and Imran Khan. This is an open access article distributed under the Creative Commons Attribution License, which permits unrestricted use, distribution, and reproduction in any medium, provided the original work is properly cited.

A collocation method based on linear Legendre multiwavelets is developed for numerical solution of one-dimensional parabolic partial integrodifferential equations of diffusion type. Such equations have numerous applications in many problems in the applied sciences to model dynamical systems. The proposed numerical method is validated by applying it to various benchmark problems from the existing literature. The numerical results confirm the accuracy, efficiency, and robustness of the proposed method.

\section{Introduction}

In some fields such as nuclear reactor dynamics and thermoelasticity we need to reflect the effect of the memory of the systems in model. If such systems are modeled using partial differential equation, which involves functions at a given space and time, the effect of past history is ignored. Therefore in order to incorporate the memory effect in such systems, an integral term in the basic partial differential equation is introduced and this leads to a partial integrodifferential equation.

Partial integrodifferential equations are used in many problems in the applied sciences to model dynamical systems. The applications of partial integrodifferential equations can be found in financial mathematics [1], biological models, chemical kinetic, aerospace systems, control theory of financial mathematics, and industrial mathematics [2]. In addition to this several phenomena in physics such as study of heat conduction, thermoelastic contact, control theory, fluid dynamics, and viscoelastic mechanics [3] can also be modeled using partial integrodifferential equation.

In this paper, we consider one-dimensional partial integrodifferential equation of diffusion type which is given as follows:

$$
\begin{aligned}
& \frac{\partial u}{\partial t}=\Delta u+h(x, t)-\int_{0}^{t} K(x, t-s) u(x, s) d s, \\
& x \in \Omega, t>0,
\end{aligned}
$$

subject to initial condition,

$$
u(x, 0)=u_{0}(x), \quad x \in \Omega,
$$

and Dirichlet boundary conditions,

$$
u(x, t)=g(x, t), \quad x \in \partial \Omega, t>0,
$$

where $\Delta u=\partial^{2} u / \partial x^{2}$ and $\partial \Omega$ is the boundary of $\Omega$. Moreover, the function $h(x, t)$ and kernel function $K(x, t)$ are assumed to be smooth and in this paper we will consider $\Omega=[0,1]$.

We note that partial integrodifferential equation of type (1) arises in several applications including analysis of spacetime-dependent nuclear reactor and blow-up problems [4].

Due to the unavailability of the analytical solution for partial integrodifferential equation, researchers have used numerical methods to find approximate solutions. Numerical solutions of integral equations, integrodifferential equations, and partial integrodifferential equations have been considered by many researchers. Several methods have been introduced including flatness method [5], curve length method [6], Tau method [7], Sobolev gradient method [8], homotopy analysis transform method [9], explicit iterative method [10-13], operational matrix method [14], homotopy analysis method [15], and Sinc collocation method [16].

Wavelets have several applications in approximation theory. A survey of some of the early works can be found 
in $[17,18]$. Wavelets have been used for numerical solutions of integral equations [19], integrodifferential equations [20], fractional diffusion-wave equation [21], ordinary differential equations [22], and partial differential equations [23]. These methods employ various types of wavelets, which include Daubechies [24], Battle-Lemarie [25], and Haar wavelet [26-28].

The rest of the paper is organized as follows. In Section 2, a brief introduction of linear Legendre multiwavelets is given. In Section 3, a solution procedure of the new method is discussed. In Section 4, numerical experiments are presented for validation of the new algorithm. Finally, in Section 5, some conclusions are drawn.

\section{The Linear Legendre Multiwavelets}

A wavelet is a class of functions constructed from dilation and translation of a single function called the mother wavelet. When the dilation and translation parameters $a$ and $b$ vary continuously, the following family of continuous wavelets is obtained:

$$
\omega_{a b}(x)=|a|^{-1 / 2} \omega\left(\frac{x-b}{a}\right), \quad a, b \in \mathbb{R}, a \neq 0,
$$

whereas if the parameters $a$ and $b$ are restricted to discrete values $a=2^{-k}$ and $b=n 2^{-k}$, then we have the following family of discrete wavelets:

$$
\omega_{k n}(x)=2^{k / 2} \omega\left(2^{k} x-n\right), \quad k, n \in \mathbb{Z},
$$

where the mother wavelet $\omega$ satisfies $\int_{\mathbb{R}} \omega(x)=0$.

In the present work only discrete wavelet family is considered. A special case where the discrete wavelet family forms an orthonormal basis of $L^{2}(\mathbb{R})$ has several advantages. This can be obtained by means of multiresolution analysis (MRA) which is defined below.

The increasing sequence $\left\{V_{k}\right\}_{k \in \mathbb{Z}}$ of closed subspaces of $L^{2}(\mathbb{R})$ with scaling function $\varphi \in V_{0}$ is called MRA if the following conditions are satisfied:

(1) $\bigcup_{k} V_{k}$ is dense in $L^{2}(\mathbb{R})$ and $\bigcap_{k} V_{k}=\{0\}$,

(2) $f(x) \in V_{k}$ iff $f\left(z^{-k} x\right) \in V_{0}$,

(3) $\{\varphi(x-n)\}_{n \in \mathbb{Z}}$ is a Riesz basis for $V_{0}$.

Note that condition (3) implies that the sequence $\left\{2^{k} \varphi\left(2^{k} x-\right.\right.$ $n)\}_{n \in \mathbb{Z}}$ is an orthonormal basis for $V_{0}$. In [29], the authors constructed the linear Legendre multiwavelets. The two scaling functions $\varphi_{0}(x)$ and $\varphi_{1}(x)$ are chosen as follows:

$$
\begin{aligned}
& \varphi_{0}(x)=1, \\
& \varphi_{1}(x)=\sqrt{3}(2 x-1), \\
& \qquad 0 \leq x<1 .
\end{aligned}
$$

Then, the corresponding mother wavelets for the linear Legendre multiwavelets family are given as follows:

$$
\omega^{0}(x)= \begin{cases}-\sqrt{3}(4 x-1) & \text { for } 0 \leq x<\frac{1}{2}, \\ \sqrt{3}(4 x-3) & \text { for } \frac{1}{2} \leq x<1,\end{cases}
$$

$$
\omega^{1}(x)= \begin{cases}(6 x-1) & \text { for } 0 \leq x<\frac{1}{2} \\ (6 x-5) & \text { for } \frac{1}{2} \leq x<1\end{cases}
$$

The linear Legendre multiwavelet is constructed by translating and dilating the mother wavelet $\omega$ and hence is given by

$$
\left\{\omega_{k n}^{j}(x)=2^{k / 2} \omega^{j}\left(2^{k} x-n\right), k, n \in \mathbb{Z}, j=0,1\right\} .
$$

The family $\left\{\omega_{k n}^{j}\right\}_{k, n \in \mathbb{Z}}^{j=0,1}$ forms an orthonormal basis for $L^{2}(\mathbb{R})$ and subfamily $\left\{\omega_{k n}^{j}\right\}$ where $n=0,1,2, \ldots, 2^{k}-1, k=0,1,2$, $\ldots$, and $j=0,1$ is an orthonormal basis for $L^{2}[0,1]$ [29]. Thus, any function $u(x) \in L^{2}[0,1]$ can be expressed as a linear combination of members of linear Legendre multiwavelets family as follows:

$$
u(x)=c_{0} \varphi_{0}(x)+c_{1} \varphi_{1}(x)+\sum_{k=0}^{\infty} \sum_{j=0}^{1} \sum_{n=0}^{2^{k}-1} c_{k n}^{j} \omega_{k n}^{j}(x),
$$

where

$$
c_{k n}^{j}=\left\langle g(x), \omega_{k n}^{j}\right\rangle
$$

and $\langle\cdot, \cdot\rangle$ denotes the standard inner product of the Hilbert space $L^{2}(\mathbb{R})$.

For approximation purposes, the infinite series in (10) is truncated, and we have

$$
u(x) \approx c_{0} \varphi_{0}(x)+c_{1} \varphi_{1}(x)+\sum_{k=0}^{M} \sum_{j=0}^{1} \sum_{n=0}^{2^{k}-1} c_{k n}^{j} \omega_{k n}^{j}(x) .
$$

The first four functions $\varphi_{0}, \varphi_{1}, \omega^{0}, \omega^{1}$ are defined earlier through (6) to (8). The next four functions are given as follows:

$$
\begin{aligned}
& \omega_{10}^{0}(x)= \begin{cases}-\sqrt{6}(8 x-1) & \text { for } 0 \leq x<\frac{1}{4}, \\
\sqrt{6}(8 x-3) & \text { for } \frac{1}{4} \leq x<\frac{1}{2}, \\
0 & \text { for } \frac{1}{2} \leq x<1,\end{cases} \\
& \omega_{11}^{0}(x)= \begin{cases}0 & \text { for } 0 \leq x<\frac{1}{2}, \\
-\sqrt{6}(8 x-5) & \text { for } \frac{1}{2} \leq x<\frac{3}{4}, \\
\sqrt{6}(8 x-7) & \text { for } \frac{3}{4} \leq x<1,\end{cases} \\
& \omega_{10}^{1}(x)= \begin{cases}\sqrt{2}(12 x-1) & \text { for } 0 \leq x<\frac{1}{4}, \\
\sqrt{2}(12 x-5) & \text { for } \frac{1}{4} \leq x<\frac{1}{2}, \\
0 & \text { for } \frac{1}{2} \leq x<1,\end{cases} \\
& \omega_{11}^{1}(x)= \begin{cases}\sqrt{2}(12 x-7) & \text { for } \frac{1}{2} \leq x<\frac{3}{4}, \\
\sqrt{2}(12 x-11) & \text { for } \frac{3}{4} \leq x<1 .\end{cases}
\end{aligned}
$$


The remaining functions can be constructed in a similar manner.

The representation of linear Legendre multiwavelets described above, that is, $\left\{\omega_{k n}^{j}: k, n \in \mathbb{Z}, j=0,1\right\}$, involves three parameters. In order to simplify the notations and reduce the parameters, we divide the linear Legendre multiwavelets

$$
\begin{aligned}
& \phi_{1}(x)=1, \quad 0 \leq x<1, \\
& \phi_{i}(x)= \begin{cases}-2^{j / 2} \sqrt{3}\left(2^{j+2} x-4 k-1\right) & \text { for } \frac{k}{m} \leq x<\frac{k+0.5}{m}, \\
2^{j / 2} \sqrt{3}\left(2^{j+2} x-4 k-3\right) & \text { for } \frac{k+0.5}{m} \leq x<\frac{k+1}{m}, i=2,3, \ldots,\end{cases}
\end{aligned}
$$

where $m=2^{j}, j=0,1, \ldots, J$, and $k=0,1, \ldots, m-1$. The integer $j$ indicates the level of resolution of the wavelet, $J$ is the maximum level of resolution, and the integer $k$ denotes the translation parameter. The relation between $m, k$, and $i$ is given as $i=m+k+1$.

$$
\begin{aligned}
& \psi_{1}(x)=\sqrt{3}(2 x-1), \quad 0 \leq x<1, \\
& \psi_{i}(x)=\left\{\begin{array}{l}
2^{j / 2}\left(3 \times 2^{j+1} x-6 k-1\right) \\
2^{j / 2}\left(3 \times 2^{j+1} x-6 k-5\right)
\end{array}\right.
\end{aligned}
$$

where $m, j$, and $k$ are defined in a similar way as above.

Using this alternate formulation of linear Legendre multiwavelets, the expression of any square integrable function $u(x)$ given in (10) can be written in an alternate form for onedimensional problem as

$$
u(x)=\sum_{i=1}^{\infty} a_{i} \phi_{i}(x)+\sum_{i=1}^{\infty} b_{i} \psi_{i}(x)
$$

and the truncated expression given in (12) becomes

$$
u(x) \approx \sum_{i=1}^{N / 2} a_{i} \phi_{i}(x)+\sum_{i=1}^{N / 2} b_{i} \psi_{i}(x)
$$

where $N=2^{J+2}$. The coefficients $a_{i}, i=1,2, \ldots, N / 2$ and $b_{i}$, $i=1,2, \ldots, N / 2$ are the linear Legendre multiwavelets coefficients. Similarly the approximation for two-dimensional problem is given as follows:

$$
\begin{aligned}
u(x, t) \approx & \sum_{i=1}^{N / 2} \sum_{j=1}^{N / 2} a_{i j} \phi_{i}(x) \phi_{j}(t) \\
& +\sum_{i=1}^{N / 2} \sum_{j=1}^{N / 2} b_{i j} \phi_{i}(x) \psi_{j}(t)
\end{aligned}
$$

family into two subfamilies each depending upon a single parameter. The first family will be represented by $\left\{\phi_{i}: i=\right.$ $1,2, \ldots\}$ and it contains the first scaling function and all other functions which are generated by the mother wavelet $\omega^{0}(t)$. This subfamily can be represented in an alternate way as follows:
The second family will be represented by $\left\{\psi_{i}: i=1,2, \ldots\right\}$ and it contains the second scaling function and all other functions which are generated by the mother wavelet $\omega^{1}(x)$. The alternate representation of this subfamily is given as follows: where $N=2^{J+2}$. The coefficients $a_{i j}, b_{i j}, c_{i j}, d_{i j}, i=1,2, \ldots$, $N / 2$, and $j=1,2, \ldots, N / 2$ are the linear Legendre multiwavelets coefficients.

The following collocation points are considered for linear Legendre multiwavelets approximations:

$$
\begin{gathered}
x_{k}=\frac{k-0.5}{N}, \quad k=1,2, \ldots, N, \\
t_{l}=\frac{l-0.5}{N}, \quad l=1,2, \ldots, N .
\end{gathered}
$$

For linear Legendre multiwavelets the following notations are introduced:

$$
\begin{aligned}
\phi_{i}^{1}(x) & =\int_{0}^{x} \phi_{i}\left(x^{\prime}\right) d x^{\prime}, \\
\phi_{i}^{v+1}(x) & =\int_{0}^{x} \phi_{i}^{v}\left(x^{\prime}\right) d x^{\prime}, \quad v=1,2 \ldots,
\end{aligned}
$$




$$
\begin{aligned}
\psi_{i}^{1}(x) & =\int_{0}^{x} \psi_{i}\left(x^{\prime}\right) d x^{\prime} \\
\psi_{i}^{v+1}(x) & =\int_{0}^{x} \psi_{i}^{v}\left(x^{\prime}\right) d x^{\prime}, \quad v=1,2 \ldots
\end{aligned}
$$

These integrals can be evaluated using the definition of linear Legendre multiwavelets in (15) and (17).

\section{Numerical Procedure}

In this section, we will first consider numerical integration using quadrature method with linear Legendre multiwavelets basis. After this, the proposed numerical method based on linear Legendre multiwavelets will be developed for partial integrodifferential equations for one-dimensional linear problems (1)-(3). We will be using collocation method and for linear Legendre multiwavelets approximations the collocation points in (21) will be considered.

3.1. Numerical Integration Using Linear Legendre Multiwavelets. Consider the integral

$$
\int_{0}^{1} u(x) d x
$$

Substituting the approximation of $u(x)$ in terms of linear Legendre multiwavelets basis given in (19), we obtain

$$
\begin{aligned}
\int_{0}^{1} u(x) d x & =\int_{0}^{1} \sum_{i=1}^{N / 2} a_{i} \phi_{i}(x) d x+\int_{0}^{1} \sum_{i=1}^{N / 2} b_{i} \psi_{i}(x) d x \\
& =a_{1},
\end{aligned}
$$

because

$$
\begin{aligned}
& \int_{0}^{1} \phi_{i}(x) d x=0, \quad \text { for } i=2,3, \ldots, \frac{N}{2} \\
& \int_{0}^{1} \psi_{i}(x) d x=0, \quad \text { for } i=1,2, \ldots, \frac{N}{2} .
\end{aligned}
$$

Hence to find the approximate value of the integral we need only to find the value of $a_{1}$. For this purpose, we put collocation points in (19) and obtain

$$
\begin{aligned}
& u\left(x_{j}\right)=\sum_{i=1}^{N / 2} a_{i} \phi_{i}\left(x_{j}\right)+\sum_{i=1}^{N / 2} b_{i} \psi_{i}\left(x_{j}\right), \\
& j=1,2, \ldots, N .
\end{aligned}
$$

Solving the above system for $a_{1}$, we get

$$
a_{1}=\frac{1}{N} \sum_{k=1}^{N} u\left(x_{k}\right) \text {. }
$$

Hence we obtain the quadrature formula for numerical integration using linear Legendre multiwavelets over the interval $[0,1]$ as follows:

$$
\int_{0}^{1} u(x) d x=\frac{1}{N} \sum_{k=1}^{N} u\left(x_{k}\right) .
$$

For a general interval $[a, b]$, we have

$$
\begin{aligned}
\int_{a}^{b} u(x) d x & =\frac{(b-a)}{N} \int_{0}^{1} u(a+(b-a) t) d t \\
& =\frac{(b-a)}{N} \sum_{k=1}^{N} u\left(t_{k}\right),
\end{aligned}
$$

where

$$
t_{k}=a+(b-a)\left(\frac{k-0.5}{N}\right), \quad k=1,2, \ldots, N
$$

3.2. One-Dimensional Partial Integrodifferential Equation. In this subsection, we will consider the application of the proposed collocation method to one-dimensional partial integrodifferential equation. For such problems the second-order partial derivative $u_{x x}(x, t)$ is approximated using two-dimensional linear Legendre multiwavelets as follows:

$$
\begin{aligned}
u_{x x}(x, t)= & \sum_{i=1}^{N / 2} \sum_{j=1}^{N / 2} a_{i j} \phi_{i}(x) \phi_{j}(t) \\
& +\sum_{i=1}^{N / 2} \sum_{j=1}^{N / 2} b_{i j} \phi_{i}(x) \psi_{j}(t) \\
& +\sum_{i=1}^{N / 2} \sum_{j=1}^{N / 2} c_{i j} \psi_{i}(x) \phi_{j}(t) \\
& +\sum_{i=1}^{N / 2} \sum_{j=1}^{N / 2} d_{i j} \psi_{i}(x) \psi_{j}(t) .
\end{aligned}
$$

Integrating (31) and using the boundary conditions in (3), we get the following expression:

$$
\begin{aligned}
u(x, t)= & \sum_{i=1}^{N / 2} \sum_{j=1}^{N / 2} a_{i j}\left(\phi_{i}^{2}(x)-x \phi_{i}^{2}(1)\right) \phi_{j}(t) \\
& +\sum_{i=1}^{N / 2} \sum_{j=1}^{N / 2} b_{i j}\left(\phi_{i}^{2}(x)-x \phi_{i}^{2}(1)\right) \psi_{j}(t) \\
& +\sum_{i=1}^{N / 2} \sum_{j=1}^{N / 2} c_{i j}\left(\psi_{i}^{2}(x)-x \psi_{i}^{2}(1)\right) \phi_{j}(t) \\
& +\sum_{i=1}^{N / 2} \sum_{j=1}^{N / 2} d_{i j}\left(\psi_{i}^{2}(x)-x \psi_{i}^{2}(1)\right) \psi_{j}(t) \\
& +g(0, t)+x(g(1, t)-g(0, t)) .
\end{aligned}
$$

In a similar manner the first-order partial derivative $u_{t}(x, t)$ is also approximated as

$$
\begin{aligned}
u_{t}(x, t)= & \sum_{i=1}^{N / 2} \sum_{j=1}^{N / 2} e_{i j} \phi_{i}(x) \phi_{j}(t) \\
& +\sum_{i=1}^{N / 2} \sum_{j=1}^{N / 2} f_{i j} \phi_{i}(x) \psi_{j}(t)
\end{aligned}
$$




$$
\begin{aligned}
& +\sum_{i=1}^{N / 2} \sum_{j=1}^{N / 2} g_{i j} \psi_{i}(x) \phi_{j}(t) \\
& +\sum_{i=1}^{N / 2} \sum_{j=1}^{N / 2} h_{i j} \psi_{i}(x) \psi_{j}(t) .
\end{aligned}
$$

Integrating (33) and using the initial condition in (2) we have

$$
\begin{aligned}
u(x, t)= & \sum_{i=1}^{N / 2} \sum_{j=1}^{N / 2} e_{i j} \phi_{i}(x) \phi_{j}^{1}(t) \\
& +\sum_{i=1}^{N / 2} \sum_{j=1}^{N / 2} f_{i j} \phi_{i}(x) \psi_{j}^{1}(t) \\
& +\sum_{i=1}^{N / 2} \sum_{j=1}^{N / 2} g_{i j} \psi_{i}(x) \phi_{j}^{1}(t) \\
& +\sum_{i=1}^{N / 2} \sum_{j=1}^{N / 2} h_{i j} \psi_{i}(x) \psi_{j}^{1}(t)+u_{0}(x) .
\end{aligned}
$$

Comparing (32) and (34) and using collocation points for $x$ and $t$ defined in (21), we obtained the following system of equations:

$$
\begin{aligned}
& \sum_{i=1}^{N / 2} \sum_{j=1}^{N / 2} a_{i j}\left(\phi_{i}^{2}\left(x_{k}\right)-x_{k} \phi_{i}^{2}(1)\right) \phi_{j}\left(t_{l}\right) \\
&+\sum_{i=1}^{N / 2} \sum_{j=1}^{N / 2} b_{i j}\left(\phi_{i}^{2}\left(x_{k}\right)-x_{k} \phi_{i}^{2}(1)\right) \psi_{j}\left(t_{l}\right) \\
&+\sum_{i=1}^{N / 2} \sum_{j=1}^{N / 2} d_{i j}\left(\psi_{i}^{2}\left(x_{k}\right)-x_{k} \psi_{i}^{2}(1)\right) \phi_{j}\left(t_{l}\right) \\
&+\sum_{i=1}^{N / 2} \sum_{j=1}^{N / 2} d_{i j}\left(\psi_{i}^{2}\left(x_{k}\right)-x_{k} \psi_{i}^{2}(1)\right) \psi_{j}\left(t_{l}\right) \\
& \quad-\sum_{i=1}^{N / 2} \sum_{j=1}^{N / 2} e_{i j} \phi_{i}\left(x_{k}\right) \phi_{j}^{1}\left(t_{l}\right) \\
& \quad-\sum_{i=1}^{N / 2} \sum_{j=1}^{N / 2} f_{i j} \phi_{i}\left(x_{k}\right) \psi_{j}^{1}\left(t_{l}\right) \\
& \quad+u_{0}\left(x_{k}\right)-g\left(0, t_{l}\right)-x\left(g\left(1, t_{l}\right)-g\left(0, t_{l}\right)\right), \\
&+ \sum_{i=1}^{N / 2} \sum_{j=1}^{N / 2} g_{i j} \psi_{i}\left(x_{k}\right) \phi_{j}^{1}\left(t_{l}\right) \\
&-\sum_{i=1}^{N / 2} \sum_{j=1}^{N / 2} h_{i j} \psi_{i}\left(x_{k}\right) \psi_{j}^{1}\left(t_{l}\right) \\
& \\
&
\end{aligned}
$$

Equation (35) represents $N \times N$ linear system with unknowns $a_{i j}, b_{i j}, c_{i j}, d_{i j}, e_{i j}, f_{i j}, g_{i j}, h_{i j}, i, j=1,2, \ldots, N / 2$.

Next we approximate the partial integrodifferential equation given in (1). Applying the numerical integration formula developed in (29), we get

$$
\begin{aligned}
u_{t}(x, t)= & u_{x x}(x, t)+h(x, t) \\
& -\frac{t}{N} \sum_{r=1}^{N} K\left(x, t-s_{r}\right) u\left(x, s_{r}\right),
\end{aligned}
$$

$$
x \in \Omega, t \in[0, T] .
$$

Substituting the values of $u_{x x}(x, t), u(x, t)$, and $u_{t}(x, t)$ from (31), (32), and (33) in (36) we have

$$
\begin{aligned}
\sum_{i=1}^{N / 2} & \sum_{j=1}^{N / 2} e_{i j} \phi_{i}(x) \phi_{j}(t)+\sum_{i=1}^{N / 2} \sum_{j=1}^{N / 2} f_{i j} \phi_{i}(x) \psi_{j}(t) \\
& +\sum_{i=1}^{N / 2} \sum_{j=1}^{N / 2} g_{i j} \psi_{i}(x) \phi_{j}(t)+\sum_{i=1}^{N / 2} \sum_{j=1}^{N / 2} h_{i j} \psi_{i}(x) \psi_{j}(t) \\
& =\sum_{i=1}^{N / 2} \sum_{j=1}^{N / 2} a_{i j} \phi_{i}(x) \phi_{j}(t)+\sum_{i=1}^{N / 2} \sum_{j=1}^{N / 2} b_{i j} \phi_{i}(x) \psi_{j}(t) \\
& +\sum_{i=1}^{N / 2} \sum_{j=1}^{N / 2} c_{i j} \psi_{i}(x) \phi_{j}(t)+\sum_{i=1}^{N / 2} \sum_{j=1}^{N / 2} d_{i j} \psi_{i}(x) \psi_{j}(t) \\
+ & h(x, t)-\frac{t}{N} \sum_{r=1}^{N} K\left(x, t-s_{r}\right) \\
+ & +\sum_{i=1}^{N / 2} \sum_{i=1}^{N / 2} a_{j=1}\left(\phi_{i}^{2}(x)-x \phi_{i}^{2}(1)\right) \phi_{j}\left(s_{r}\right) \\
+ & \sum_{i=1}^{N / 2} \sum_{j=1}^{N / 2} c_{i j}\left(\psi_{i}^{2}(x)-x \psi_{i}^{2}(1)\right) \phi_{j}\left(s_{r}\right) \\
+ & \sum_{i=1}^{N / 2} b_{i j}\left(\phi_{i}^{2}(x)-x \phi_{i}^{2}(1)\right) \psi_{j}\left(s_{r}\right) \\
& \left.\left.+x / 2)-g\left(0, s_{r}\right)\right) s_{i}^{2}(1)\right) \psi_{j}\left(s_{r}\right)+g\left(0, s_{r}\right) \\
+ &
\end{aligned}
$$

Using collocation points for $x$ and $t$ defined in (21), we have the following system of equations after simplification:

$$
\begin{gathered}
\sum_{i=1}^{N / 2} \sum_{j=1}^{N / 2} a_{i j}\left(\frac{t}{N} \sum_{r=1}^{N} K\left(x_{k}, t_{l}-s_{r}\right)\left(\phi_{i}^{2}\left(x_{k}\right)-x_{k} \phi_{i}^{2}(1)\right)\right. \\
\left.\cdot \phi_{j}\left(s_{r}\right)-\phi_{i}\left(x_{k}\right) \phi_{j}\left(t_{l}\right)\right)+\sum_{i=1}^{N / 2} \sum_{j=1}^{N / 2} b_{i j}\left(\frac{t}{N}\right.
\end{gathered}
$$




$$
\begin{aligned}
& \cdot \sum_{r=1}^{N} K\left(x_{k}, t_{l}-s_{r}\right)\left(\phi_{i}^{2}\left(x_{k}\right)-x_{k} \phi_{i}^{2}(1)\right) \psi_{j}\left(s_{r}\right) \\
& \left.-\phi_{i}\left(x_{k}\right) \psi_{j}\left(t_{l}\right)\right)+\sum_{i=1}^{N / 2} \sum_{j=1}^{N / 2} c_{i j}\left(\frac{t}{N} \sum_{r=1}^{N} K\left(x_{k}, t_{l}-s_{r}\right)\right. \\
& \left.\cdot\left(\psi_{i}^{2}\left(x_{k}\right)-x_{k} \psi_{i}^{2}(1)\right) \phi_{j}\left(s_{r}\right)-\psi_{i}\left(x_{k}\right) \phi_{j}\left(t_{l}\right)\right) \\
& +\sum_{i=1}^{N / 2} \sum_{j=1}^{N / 2} d_{i j}\left(\frac{t}{N} \sum_{r=1}^{N} K\left(x_{k}, t_{l}-s_{r}\right)\right. \\
& \left.\cdot\left(\psi_{i}^{2}\left(x_{k}\right)-x_{k} \psi_{i}^{2}(1)\right) \psi_{j}\left(s_{r}\right)-\phi_{i}\left(x_{k}\right) \psi_{j}\left(t_{l}\right)\right) \\
& +\sum_{i=1}^{N / 2} \sum_{j=1}^{N / 2} e_{i j} \phi_{i}\left(x_{k}\right) \phi_{j}\left(t_{l}\right)+\sum_{i=1}^{N / 2} \sum_{j=1}^{N / 2} f_{i j} \phi_{i}\left(x_{k}\right) \psi_{j}\left(t_{l}\right) \\
& +\sum_{i=1}^{N / 2} \sum_{j=1}^{N / 2} g_{i j} \psi_{i}\left(x_{k}\right) \phi_{j}\left(t_{l}\right) \\
& +\sum_{i=1}^{N / 2} \sum_{j=1}^{N / 2} h_{i j} \psi_{i}\left(x_{k}\right) \psi_{j}\left(t_{l}\right)=\frac{t}{N} \sum_{r=1}^{N} K\left(x_{k}, t_{l}-s_{r}\right) \\
& \cdot\left(x\left(g\left(0, s_{r}\right)-g\left(1, s_{r}\right)\right)-g\left(0, s_{r}\right)\right) \\
& +h\left(x_{k}, t_{l}\right) \quad \text { for } k, l=1,2, \ldots, N \text {. }
\end{aligned}
$$

Again we obtain an $N \times N$ linear system with unknowns $a_{i j}, b_{i j}, c_{i j}, d_{i j}, e_{i j}, f_{i j}, g_{i j}, h_{i j}, i, j=1,2, \ldots, N / 2$.

Finally these two systems in (35) and (38) can be solved easily by Gauss elimination method. The solution of these two systems gives the values of the unknown coefficients $a_{i j}, b_{i j}, c_{i j}$, $d_{i j}, e_{i j}, f_{i j}, g_{i j}, h_{i j}, i, j=1,2, \ldots, N / 2$ at the collocation points defined in (21). With these coefficients we approximate $u(x$, $t$ ) which is the solution of the partial integrodifferential equation in (1).

\section{Numerical Experiments}

In this section, several numerical experiments are performed in order to show the performance of the proposed method. The method is applied to six linear one-dimensional partial integrodifferential equations (Test Problems 1-6).

Test Problem 1. Consider a diffusion problem [30]:

$$
\begin{aligned}
& \frac{\partial u}{\partial t}=\frac{\partial^{2} u}{\partial x^{2}}-\int_{0}^{t} e^{(x(t-s))} u(x, s) d s+ h(x, t), \\
& x \in \Omega, t \in[0,1],
\end{aligned}
$$

subject to the following initial and boundary conditions:

$$
\begin{aligned}
& u(x, 0)=0, \\
& u(0, t)=\sin t,
\end{aligned}
$$

$$
\begin{aligned}
& u(1, t)=0, \\
& u(x, t)=\left(1-x^{2}\right) \sin t
\end{aligned}
$$

as the exact solution of problem (1)-(3), where $h(x, t)$ is given as

$$
\begin{aligned}
h(x, t)= & \left(1-x^{2}\right) \cos t+2 \sin t \\
& +\frac{\left(x^{2}-1\right)\left(\cos t+x \sin t-e^{x t}\right)}{1+x^{2}} .
\end{aligned}
$$

Two-dimensional linear Legendre multiwavelets approximation is applied to this test problem and numerical results at different time levels are reported in Table 1. From this table one can easily observe that the maximum absolute errors are decreasing by increasing the number of collocation points. An accuracy of order $10^{-5}$ is obtained by considering $N=$ $32 \times 32$ number of collocation points. Note that since this is not time marching scheme, therefore the maximum absolute errors are not increased while moving with time. Instead, we observe an oscillating behavior in maximum absolute errors for fixed number of collocation points; for some time steps they are increasing whereas for other time levels they are decreasing.

We have also plotted the graphs of exact and approximate solutions in Figure 1 for comparison. The graph of approximate solution is plotted using $N=32 \times 32$ collocation points and it is in complete agreement with the graph of exact solution.

Test Problem 2. Consider a linear diffusion equation [30]:

$$
\begin{array}{r}
\frac{\partial u}{\partial t}=\frac{\partial^{2} u}{\partial x^{2}}-\int_{0}^{t} \sin x(t-s) u(x, s) d s+h(x, t), \\
x \in \Omega, t \in[0,1],
\end{array}
$$

subject to initial and boundary conditions:

$$
\begin{aligned}
& u(x, 0)=1-x^{2}, \\
& u(0, t)=e^{t} \\
& u(1, t)=0 .
\end{aligned}
$$

The exact solution of this problem is as follows:

$$
u(x, t)=\left(1-x^{2}\right) e^{t}
$$

where the function $h(x, t)$ is calculated using the above exact solution as follows:

$$
\begin{aligned}
h(x, t)= & 2 e^{t}+e^{t}\left(1-x^{2}\right) \\
& +\frac{\left(-1+x^{2}\right)\left(-e^{t} x+x \cos t x+\sin t x\right)}{1+x^{2}} .
\end{aligned}
$$

The exact solution of this test problem involves trigonometric functions. Numerical results using two-dimensional Haar 
TABLE 1: Maximum absolute errors at different time of the present method for Test Problem 1.

\begin{tabular}{lcccc}
\hline$t$ & $N=4 \times 4$ & $N=8 \times 8$ & $N=16 \times 16$ & $N=32 \times 32$ \\
\hline 0.0625 & $1.0278 \times 10^{-3}$ & $7.4383 \times 10^{-5}$ & $4.6240 \times 10^{-6}$ & $1.2106 \times 10^{-5}$ \\
0.1875 & $1.8960 \times 10^{-4}$ & $7.5155 \times 10^{-5}$ & $1.2275 \times 10^{-5}$ & $2.4685 \times 10^{-5}$ \\
0.3125 & $7.0791 \times 10^{-4}$ & $1.4643 \times 10^{-4}$ & $2.5696 \times 10^{-5}$ & $3.5745 \times 10^{-5}$ \\
0.4375 & $1.5070 \times 10^{-4}$ & $7.5929 \times 10^{-5}$ & $4.2169 \times 10^{-5}$ & $4.5563 \times 10^{-5}$ \\
0.5625 & $2.6171 \times 10^{-3}$ & $1.2180 \times 10^{-4}$ & $6.0743 \times 10^{-5}$ & $5.3926 \times 10^{-5}$ \\
0.6875 & $7.2426 \times 10^{-4}$ & $1.0567 \times 10^{-4}$ & $8.1933 \times 10^{-5}$ & $6.0499 \times 10^{-5}$ \\
0.8125 & $1.5578 \times 10^{-3}$ & $4.7215 \times 10^{-5}$ & $1.0738 \times 10^{-4}$ & $6.4915 \times 10^{-5}$ \\
0.9375 & $4.2985 \times 10^{-4}$ & $2.1869 \times 10^{-4}$ & $1.3833 \times 10^{-4}$ & $6.6396 \times 10^{-5}$ \\
\hline
\end{tabular}

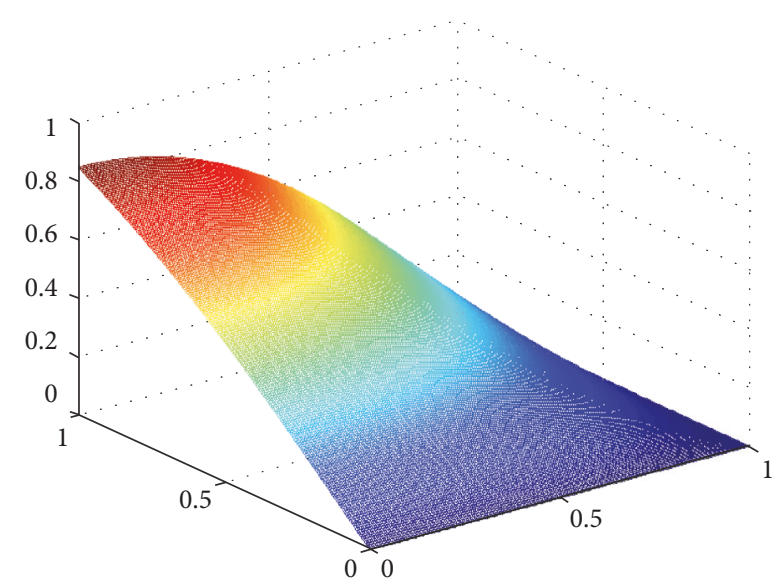

Exact solution

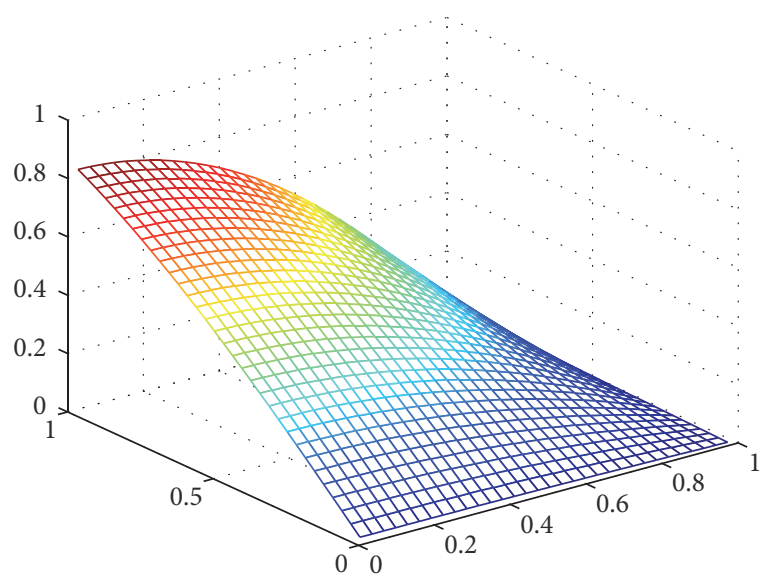

Approximate solution $(N=32 \times 32)$

Figure 1: Plots of exact and approximate solution for Test Problem 1.

wavelet in terms of maximum absolute errors at different time levels are reported in Table 2. The table shows a consistent behavior of the proposed method as the maximum absolute errors increase with increase in number of collocation points.

Test Problem 3. In this example [30], we investigate a linear diffusion problem as

$$
\begin{array}{r}
\frac{\partial u}{\partial t}=\frac{\partial^{2} u}{\partial x^{2}}-\int_{0}^{t} \frac{t-s+1}{x+1} u(x, s) d s+h(x, t), \\
x \in \Omega, t \in[0,1],
\end{array}
$$

subject to initial and boundary conditions:

$$
\begin{aligned}
& u(x, 0)=1-x^{2}, \\
& u(0, t)=\frac{1}{1+t^{2}}, \\
& u(1, t)=0,
\end{aligned}
$$

with the exact solution

$$
u(x, t)=\frac{1-x^{2}}{1+t^{2}},
$$

and $h(x, t)$ is defined as

$$
\begin{aligned}
& h(x, t) \\
& =\frac{2}{1+t^{2}}-\frac{2 t\left(1-x^{2}\right)}{\left(1+t^{2}\right)^{2}} \\
& \quad+\frac{(x-1)\left(-2(1+t) \tan ^{-1}(t)+\log \left(1+t^{2}\right)\right)}{2} .
\end{aligned}
$$

We have applied the proposed method to this linear test problem and the maximum absolute errors at different time levels are shown in Table 3. Once again a very good performance of the proposed method is observed from this table.

Test Problem 4. Consider another linear diffusion equation [30]:

$$
\frac{\partial u}{\partial t}=\frac{\partial^{2} u}{\partial x^{2}}-\int_{0}^{t} u(x, s) d s+h(x, t)
$$


TABLE 2: Maximum absolute errors at different time of the present method for Test Problem 2.

\begin{tabular}{lcccc}
\hline$t$ & $N=4 \times 4$ & $N=8 \times 8$ & $N=16 \times 16$ & $N=32 \times 32$ \\
\hline 0.03125 & $5.3955 \times 10^{-3}$ & $7.8261 \times 10^{-4}$ & $1.3271 \times 10^{-5}$ & $3.0776 \times 10^{-6}$ \\
0.15625 & $7.2584 \times 10^{-4}$ & $4.5256 \times 10^{-4}$ & $2.7204 \times 10^{-5}$ & $3.2693 \times 10^{-6}$ \\
0.28125 & $2.4940 \times 10^{-3}$ & $1.1149 \times 10^{-3}$ & $3.4060 \times 10^{-5}$ & $1.6258 \times 10^{-5}$ \\
0.40625 & $7.3514 \times 10^{-4}$ & $5.31440 \times 10^{-4}$ & $4.0383 \times 10^{-5}$ & $2.0544 \times 10^{-5}$ \\
0.53125 & $1.0091 \times 10^{-2}$ & $1.4578 \times 10^{-3}$ & $4.8419 \times 10^{-5}$ & $1.5133 \times 10^{-5}$ \\
0.65625 & $4.5156 \times 10^{-4}$ & $6.4253 \times 10^{-4}$ & $5.9778 \times 10^{-5}$ & $4.9830 \times 10^{-6}$ \\
0.78125 & $3.5433 \times 10^{-3}$ & $1.9173 \times 10^{-3}$ & $7.5454 \times 10^{-5}$ & $3.0333 \times 10^{-5}$ \\
0.90625 & $1.6591 \times 10^{-3}$ & $7.3846 \times 10^{-4}$ & $9.6496 \times 10^{-5}$ & $6.3594 \times 10^{-6}$ \\
\hline
\end{tabular}

TABle 3: Maximum absolute errors at different time of the present method for Test Problem 3.

\begin{tabular}{lcccc}
\hline$t$ & $N=4 \times 4$ & $N=8 \times 8$ & $N=16 \times 16$ & $N=32 \times 32$ \\
\hline 0.1 & $3.7913 \times 10^{-4}$ & $7.8437 \times 10^{-4}$ & $1.1785 \times 10^{-4}$ & $4.9833 \times 10^{-5}$ \\
0.2 & $2.3940 \times 10^{-3}$ & $6.0770 \times 10^{-4}$ & $1.7295 \times 10^{-4}$ & $6.2964 \times 10^{-6}$ \\
0.3 & $9.8721 \times 10^{-4}$ & $5.2793 \times 10^{-4}$ & $1.8362 \times 10^{-4}$ & $1.0686 \times 10^{-5}$ \\
0.4 & $3.5740 \times 10^{-3}$ & $3.3500 \times 10^{-4}$ & $1.0876 \times 10^{-4}$ & $3.5224 \times 10^{-5}$ \\
0.5 & $7.0464 \times 10^{-3}$ & $1.0127 \times 10^{-3}$ & $1.2916 \times 10^{-4}$ & $1.5531 \times 10^{-5}$ \\
0.6 & $4.2963 \times 10^{-3}$ & $3.7386 \times 10^{-4}$ & $3.8243 \times 10^{-5}$ & $1.1223 \times 10^{-5}$ \\
0.7 & $1.6758 \times 10^{-3}$ & $2.3956 \times 10^{-4}$ & $1.4320 \times 10^{-5}$ & $1.8332 \times 10^{-5}$ \\
0.8 & $3.8863 \times 10^{-4}$ & $4.1881 \times 10^{-4}$ & $1.2823 \times 10^{-5}$ & $1.1718 \times 10^{-5}$ \\
0.9 & $1.3031 \times 10^{-3}$ & $1.1242 \times 10^{-4}$ & $5.0721 \times 10^{-5}$ & $7.1565 \times 10^{-6}$ \\
1.0 & $1.6426 \times 10^{-3}$ & $6.2199 \times 10^{-4}$ & $1.7152 \times 10^{-4}$ & $4.2592 \times 10^{-5}$ \\
\hline
\end{tabular}

subject to the following initial and boundary conditions:

$$
\begin{aligned}
& u(x, 0)=\frac{1-x^{2}}{2}, \\
& u(0, t)=\frac{\cosh t}{2+\sinh ^{2} t} \\
& u(1, t)=0
\end{aligned}
$$

The exact solution of the problem is given by

$$
u(x, t)=\frac{\left(1-x^{2}\right) \cosh t}{2+\sinh ^{2} t}
$$

and the function $h(x, t)$ is calculated using the exact solution of this test problem given as

$$
\begin{aligned}
h(x, t)= & \frac{\left(1-x^{2}\right) \sinh t+2 \cosh t}{2+\sinh t^{2}} \\
& -\frac{2\left(1-x^{2}\right) \cosh t^{2} \sinh t}{\left(2+\sinh t^{2}\right)^{2}} \\
& +\frac{1}{\sqrt{2}} \tan ^{-1}\left(\frac{1}{\sqrt{2}}\right) \sinh t\left(1-x^{2}\right) .
\end{aligned}
$$

The exact solution of this test problem involves hyperbolic functions. Two-dimensional linear Legendre multiwavelets approximation is also applied to this test problem and numerical results at different time levels are reported in Table 4.
From this table we also observe that the maximum absolute errors are decreasing by increasing the number of collocation points. An accuracy of order $10^{-6}$ is obtained by considering $N=32 \times 32$ number of collocation points.

Test Problem 5. We now turn to another example [30] of linear diffusion equation:

$$
\begin{array}{r}
\frac{\partial u}{\partial t}=\frac{\partial^{2} u}{\partial x^{2}}-\int_{0}^{t} \cos (x(t-s)) u(x, s) d s+h(x, t), \\
x \in \Omega, t \in[0,1],
\end{array}
$$

subject to the following initial and boundary conditions:

$$
\begin{aligned}
& u(x, 0)=\left(1-x^{4}\right) e^{x}, \\
& u(0, t)=e^{t} \\
& u(1, t)=0,
\end{aligned}
$$

and the exact solution is as follows:

$$
u(x, t)=\left(1-x^{4}\right) e^{x+t}
$$

followed by $h(x, t)$; that is,

$$
\begin{aligned}
& h(x, t)=e^{x}\left(e^{t}\left(1+x^{2}(11+8 x)\right)\right. \\
& \left.\quad+\left(x^{2}-1\right)(\cos (t x)-x \sin (t x))\right)
\end{aligned}
$$

This problem is solved using the two-dimensional linear Legendre multiwavelets and the numerical results are depicted in 
TABLE 4: Maximum absolute errors at different time of the present method for Test Problem 4.

\begin{tabular}{lccccc}
\hline$t$ & $N=4 \times 4$ & $N=8 \times 8$ & $N=16 \times 16$ & $N=32 \times 32$ & $N=64 \times 64$ \\
\hline 0.1 & $1.3938 \times 10^{-4}$ & $1.8049 \times 10^{-6}$ & $9.2110 \times 10^{-7}$ & $9.5195 \times 10^{-8}$ & $1.1342 \times 10^{-7}$ \\
0.2 & $5.5810 \times 10^{-5}$ & $1.3464 \times 10^{-5}$ & $2.3295 \times 10^{-6}$ & $1.5485 \times 10^{-6}$ & $4.3291 \times 10^{-8}$ \\
0.3 & $1.8068 \times 10^{-4}$ & $7.2956 \times 10^{-5}$ & $1.9806 \times 10^{-6}$ & $1.0885 \times 10^{-6}$ & $1.8598 \times 10^{-7}$ \\
0.4 & $1.5749 \times 10^{-4}$ & $4.4007 \times 10^{-5}$ & $1.7830 \times 10^{-5}$ & $1.2562 \times 10^{-6}$ & $5.6575 \times 10^{-7}$ \\
0.5 & $1.8056 \times 10^{-3}$ & $3.7671 \times 10^{-4}$ & $7.7404 \times 10^{-5}$ & $1.7270 \times 10^{-5}$ & $4.0517 \times 10^{-6}$ \\
0.6 & $5.8196 \times 10^{-4}$ & $4.5192 \times 10^{-5}$ & $9.5238 \times 10^{-6}$ & $3.7954 \times 10^{-6}$ & $1.0850 \times 10^{-6}$ \\
0.7 & $2.1447 \times 10^{-4}$ & $2.3648 \times 10^{-5}$ & $2.0197 \times 10^{-5}$ & $4.6581 \times 10^{-6}$ & $1.1987 \times 10^{-6}$ \\
0.8 & $4.2761 \times 10^{-4}$ & $8.3275 \times 10^{-5}$ & $2.3185 \times 10^{-5}$ & $3.4690 \times 10^{-6}$ & $1.5333 \times 10^{-6}$ \\
0.9 & $1.0542 \times 10^{-4}$ & $1.0790 \times 10^{-4}$ & $1.1975 \times 10^{-5}$ & $7.1800 \times 10^{-6}$ & $7.5777 \times 10^{-7}$ \\
1.0 & $1.0145 \times 10^{-3}$ & $3.4302 \times 10^{-4}$ & $8.6424 \times 10^{-5}$ & $2.0314 \times 10^{-5}$ & $4.5429 \times 10^{-6}$ \\
\hline
\end{tabular}

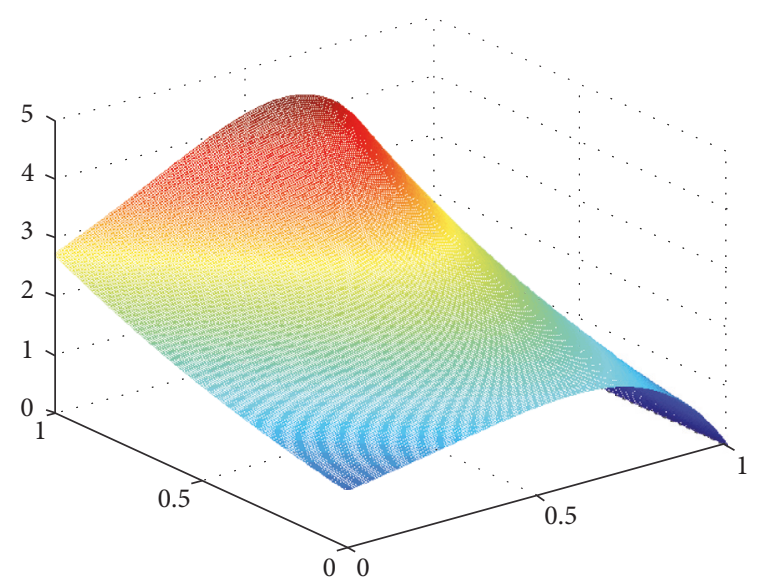

Exact solution

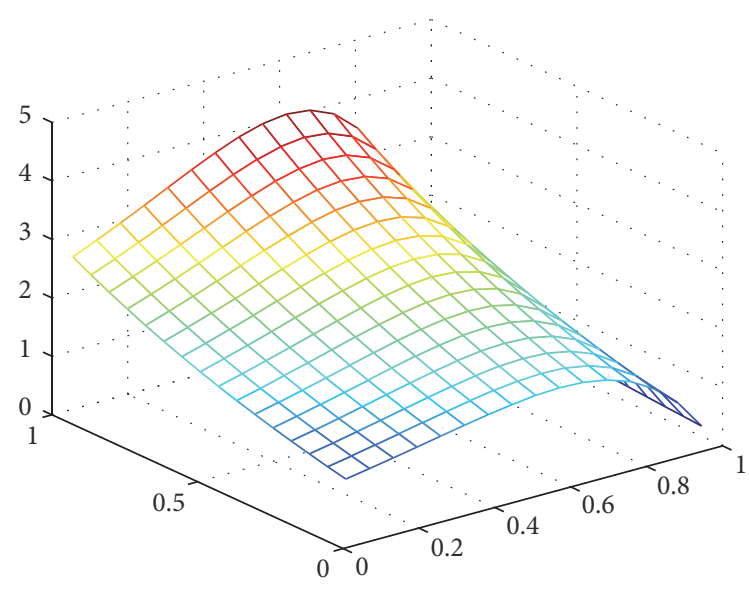

Approximate solution $(N=16 \times 16)$

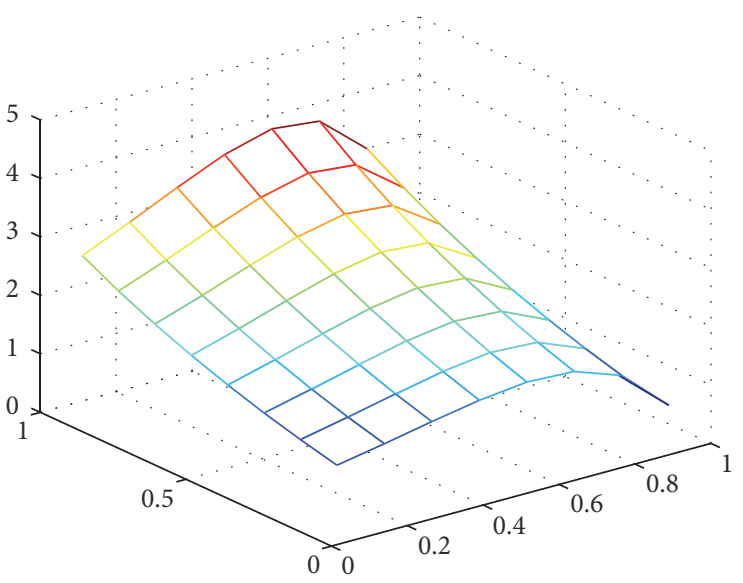

Approximate solution $(N=8 \times 8)$

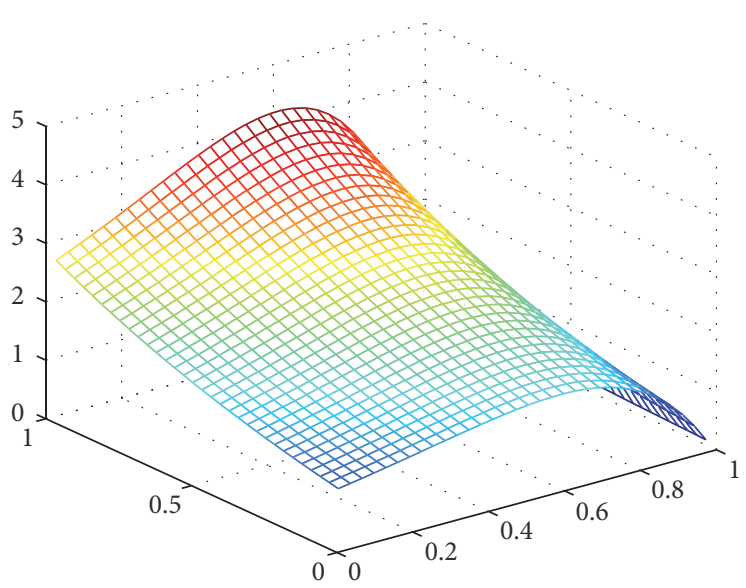

Approximate solution $(N=32 \times 32)$

FIgURE 2: Plots of exact and approximate solution for Test Problem 5.

Figure 2. In this figure we have plotted the three-dimensional graphs of the approximate solutions for $0<x<1$ and $0 \leq t \leq$ 1 using three different numbers of collocation points. Moreover for comparison purpose we have also plotted the exact solution. One can easily observe from the figure that the approximate solution is converging rapidly towards the exact solution by increasing the number of collocation points.
Test Problem 6. As a last example of the diffusion equation [30] for the linear case, we have

$$
\frac{\partial u}{\partial t}=\frac{\partial^{2} u}{\partial x^{2}}-\int_{0}^{t} u(x, s) d s+h(x, t),
$$

$x \in \Omega, t \in[0,1]$ 


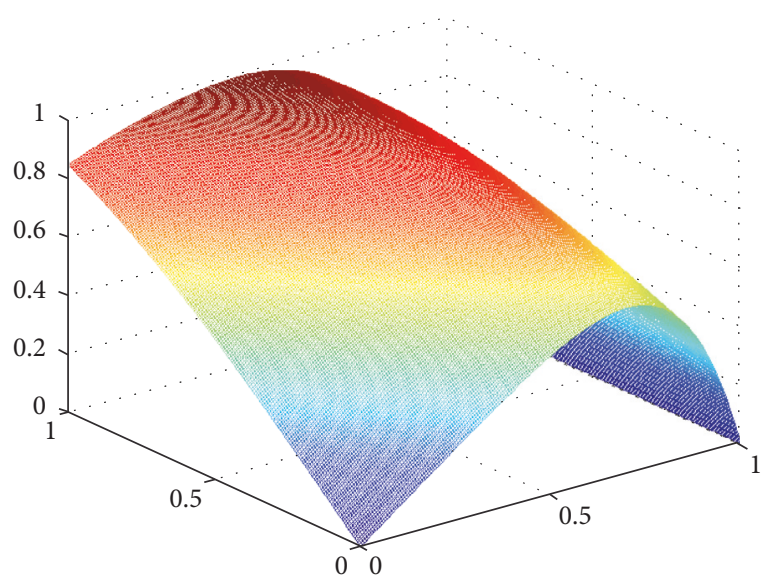

Exact solution

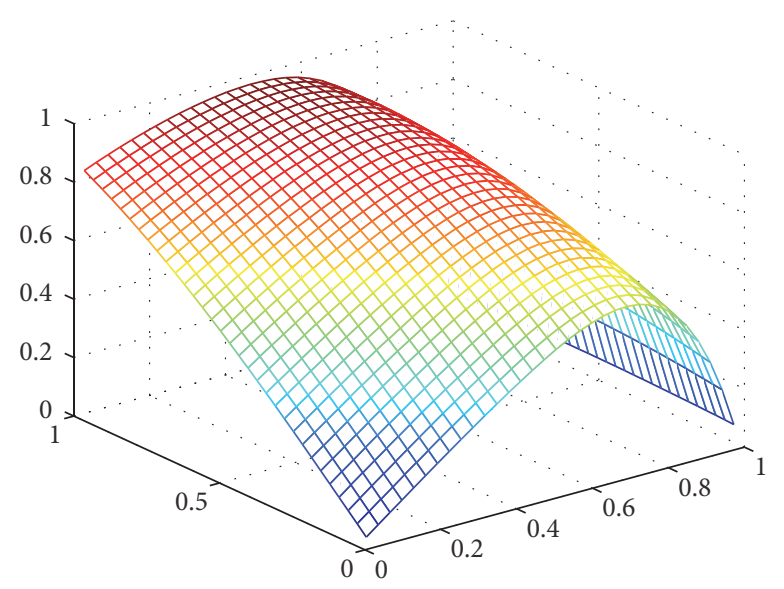

Approximate solution $(N=32 \times 32)$

FIgure 3: Plots of exact and approximate solution for Test Problem 6.

subject to initial and boundary conditions

$$
\begin{aligned}
& u(x, 0)=\left(1-x^{6}\right) \sin x \\
& u(0, t)=\sin t \\
& u(1, t)=0 .
\end{aligned}
$$

The exact solution of this two-dimensional partial integrodifferential equation is

$$
u(x, t)=\left(1-x^{6}\right) \sin (x+t)
$$

whereas the function $h(x, t)$ is calculated using the above exact solution given by

$$
\begin{aligned}
h(x, t)= & \left(1-x^{6}\right) \cos x+12 x^{5} \cos (t+x) \\
& +\left(1+30 x^{4}-x^{6}\right) \sin (t+x) .
\end{aligned}
$$

The comparison of the exact and approximate solution at the final time $t=1$ is depicted in Figure 3. This figure shows that the approximate solutions for just $N=32 \times 32$ number of collocation points are in complete agreement with the exact solution.

\section{Conclusion}

A new algorithm is proposed for the numerical solution of diffusion partial integrodifferential equation arising in the applied sciences to model dynamical systems. A two-dimensional linear Legendre multiwavelets basis is used for this purpose. The algorithm is established theoretically and, finally, we demonstrate the efficiency and accuracy of the proposed method with linear test problems.

\section{Conflicts of Interest}

The authors declare that they have no conflicts of interest.

\section{References}

[1] F. Shakeri and M. Dehghan, "A high order finite volume element method for solving elliptic partial integro-differential equations," Applied Numerical Mathematics, vol. 65, pp. 105-118, 2013.

[2] M. Dehghan and R. Salehi, "The numerical solution of the non-linear integro-differential equations based on the meshless method," Journal of Computational and Applied Mathematics, vol. 236, no. 9, pp. 2367-2377, 2012.

[3] M. Dehghan, "Solution of a partial integro-differential equation arising from viscoelasticity," International Journal of Computer Mathematics, vol. 83, no. 1, pp. 123-129, 2006.

[4] E. G. Yanik and G. Fairweather, "Finite element methods for parabolic and hyperbolic partial integro-differential equations," Nonlinear Analysis. Theory, Methods \& Applications. An International Multidisciplinary Journal, vol. 12, no. 8, pp. 785-809, 1988.

[5] T. Meurer, "Flatness of a class of linear Volterra partial integrodifferential equations," IFAC-PapersOnLine, vol. 49, no. 8, pp. 174-179, 2016.

[6] Y. Z. Chen, X. Y. Lin, Z. X. Wang, and N. M. A. Nik Long, "Solution of contact problem for an arc crack using hypersingular integral equation," International Journal of Computational Methods, vol. 5, no. 1, pp. 119-133, 2008.

[7] S. M. Hosseini and S. Shahmorad, "Numerical solution of a class of integro-differential equations by the tau method with an error estimation," Applied Mathematics and Computation, vol. 136, no. 2-3, pp. 559-570, 2003.

[8] N. Raza, S. Sial, J. W. Neuberger, and M. O. Ahmad, "Numerical solutions of integro-differential equations using Sobolev gradient methods," International Journal of Computational Methods, vol. 9, no. 4, Article ID 1250046, 2012.

[9] S. Kumar, "A new analytical modelling for fractional telegraph equation via Laplace transform," Applied Mathematical Modelling, vol. 38, no. 13, pp. 3154-3163, 2014.

[10] N. Jha, R. K. Mohanty, and B. K. Mishra, "Alternating group explicit iterative method for nonlinear singular Fredholm integro-differential boundary value problems," International Journal of Computer Mathematics, vol. 86, no. 9, pp. 1645-1656, 2009. 
[11] R. K. Mohanty and D. Dhall, "Third order accurate variable mesh discretization and application of TAGE iterative method for the non-linear two-point boundary value problems with homogeneous functions in integral form," Applied Mathematics and Computation, vol. 215, no. 6, pp. 2024-2034, 2009.

[12] R. K. Mohanty, M. K. Jain, and D. Dhall, "A cubic spline approximation and application of TAGE iterative method for the solution of two point boundary value problems with forcing function in integral form," Applied Mathematical Modelling, vol. 35, no. 6, pp. 3036-3047, 2011.

[13] R. K. Mohanty, "A combined arithmetic average discretization and TAGE iterative method for non-linear two point boundary value problems with a source function in integral form," Differential Equations and Dynamical Systems, vol. 20, no. 4, pp. 423-440, 2012.

[14] S. Shahmorad and M. H. Ostadzad, "An Operational Matrix Method for Solving Delay Fredholm and Volterra IntegroDifferential Equations," International Journal of Computational Methods, vol. 13, no. 6, Article ID 1650040, 2016.

[15] A. Shidfar, A. Molabahrami, A. Babaei, and A. Yazdanian, "A series solution of the nonlinear Volterra and Fredholm integrodifferential equations," Communications in Nonlinear Science and Numerical Simulation, vol. 15, no. 2, pp. 205-215, 2010.

[16] M. Zarebnia, "Sinc numerical solution for the Volterra integrodifferential equation," Communications in Nonlinear Science and Numerical Simulation, vol. 15, no. 3, pp. 700-706, 2010.

[17] B. Li, X. Chen, and Z. He, "A wavelet-based error estimator and an adaptive scheme for plate bending problems," International Journal of Computational Methods, vol. 7, no. 2, pp. 241-259, 2010.

[18] W. Dahmen, A. Kurdila, and P. Oswald, Multiscale Wavelet Methods for Partial Differential Equations, Academic Press, 1997.

[19] I. Aziz and S. Islam, "New algorithms for the numerical solution of nonlinear Fredholm and Volterra integral equations using Haar wavelets," Journal of Computational and Applied Mathematics, vol. 239, pp. 333-345, 2013.

[20] M. H. Heydari, M. R. Hooshmandasl, F. Mohammadi, and C. Cattani, "Wavelets method for solving systems of nonlinear singular fractional Volterra integro-differential equations," Communications in Nonlinear Science and Numerical Simulation, vol. 19, no. 1, pp. 37-48, 2014.

[21] M. H. Heydari, M. R. Hooshmandasl, F. Mohammadi, and C. Cattani, "Wavelets method for the time fractional diffusionwave equation," Physics Letters A, vol. 379, pp. 71-76, 2015.

[22] S. Islam, I. Aziz, and B. Sarler, "The numerical solution of second-order boundary-value problems by collocation method with the Haar wavelets," Mathematical and Computer Modelling, vol. 5, pp. 1577-1590, 2010.

[23] V. Comincioli, G. Naldi, and T. Scapolla, "Wavelet-based method for numerical solution of nonlinear evolution equations," Applied Numerical Mathematics, vol. 33, no. 1, pp. 291297,2000

[24] L. A. Díaz, M. T. Martín, and V. Vampa, "Daubechies wavelet beam and plate finite elements," Finite Elements in Analysis and Design, vol. 45, no. 3, pp. 200-209, 2009.

[25] X. Zhu, G. Lei, and G. Pan, "On application of fast and adaptive periodic Battle-Lemarie wavelets to modeling of multiple lossy transmission lines," Journal of Computational Physics, vol. 132, no. 2, pp. 299-311, 1997.
[26] J. Majak, B. S. Shvartsman, M. Kirs, M. Pohlak, and H. Herranen, "Convergence theorem for the haar wavelet based discretization method," Composite Structures, vol. 126, pp. 227-232, 2015.

[27] J. Majak, B. Shvartsman, K. Karjust, M. Mikola, A. Haavajõe, and M. Pohlak, "On the accuracy of the Haar wavelet discretization method," Composites Part B: Engineering, vol. 80, pp. 321$327,2015$.

[28] U. Lepik and H. Hein, Haar Wavelets with Applications, Springer, 2014.

[29] V. K. Singh, O. P. Singh, and R. K. Pandey, "Numerical evaluation of the Hankel transform by using linear Legendre multiwavelets," Computer Physics Communications, vol. 179, no. 6, pp. 424-429, 2008.

[30] F. Fakhar-Izadi and M. Dehghan, "The spectral methods for parabolic Volterra integro-differential equations," Journal of Computational and Applied Mathematics, vol. 235, no. 14, pp. 4032-4046, 2011. 


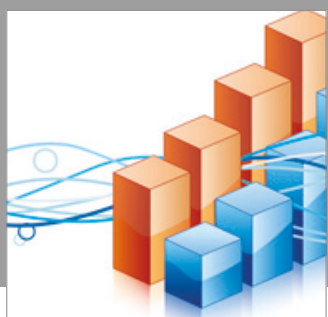

Advances in

Operations Research

vatersals

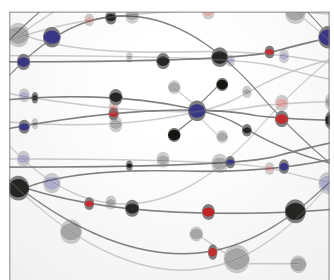

\section{The Scientific} World Journal
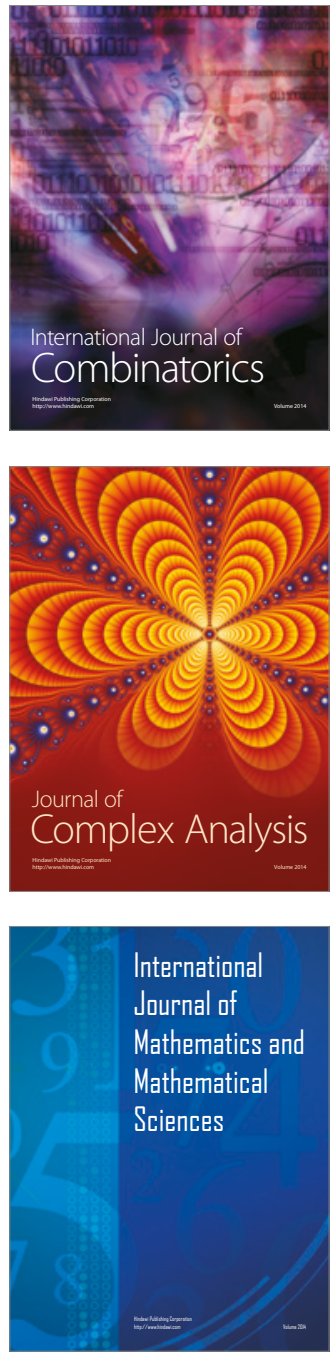
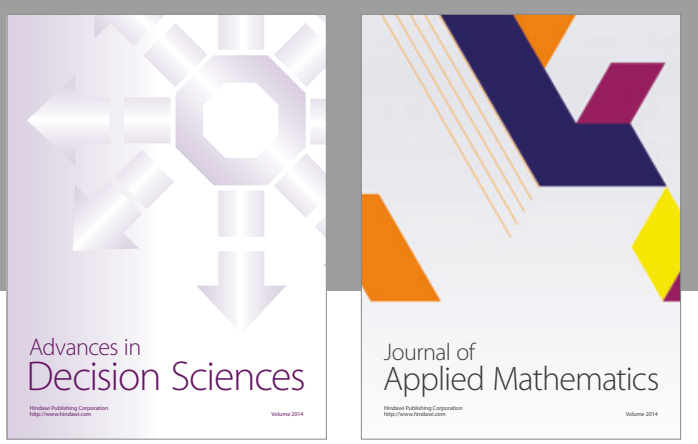

Algebra

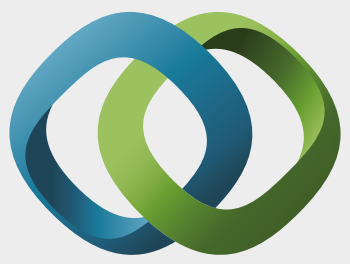

\section{Hindawi}

Submit your manuscripts at

https://www.hindawi.com
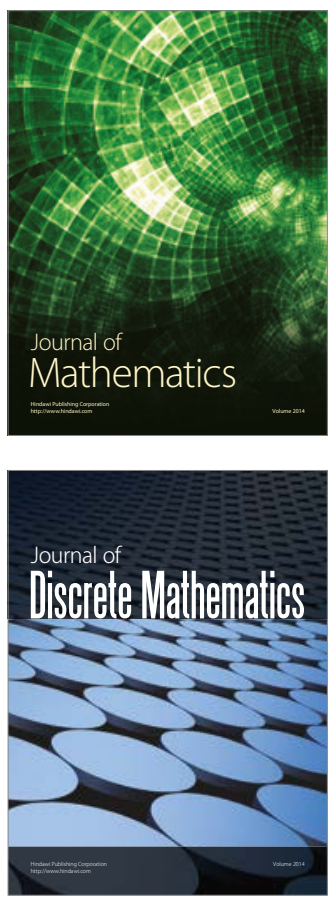

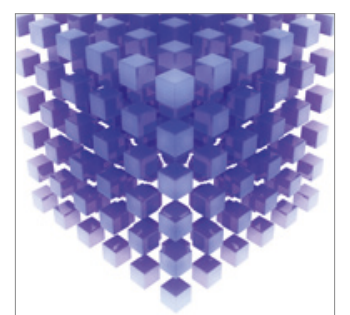

Mathematical Problems in Engineering
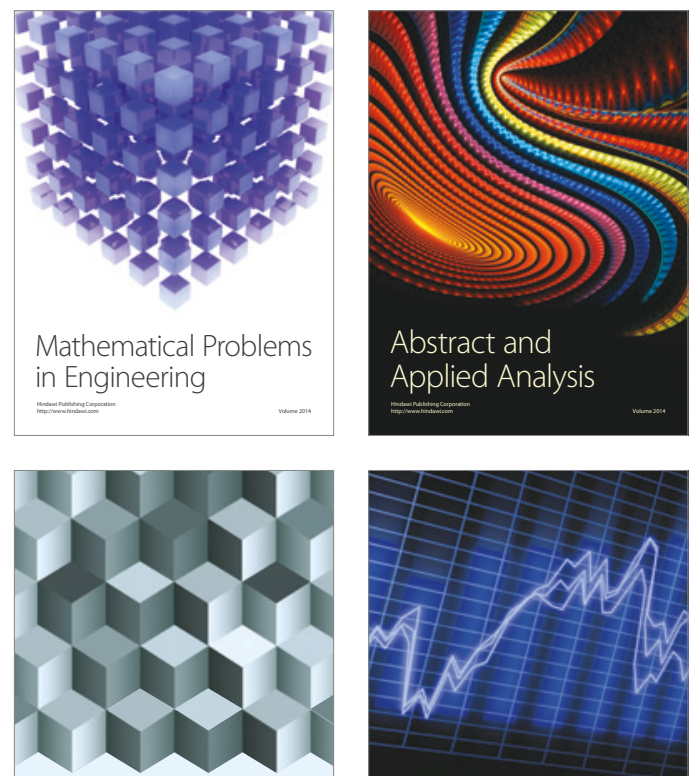

Journal of

Function Spaces

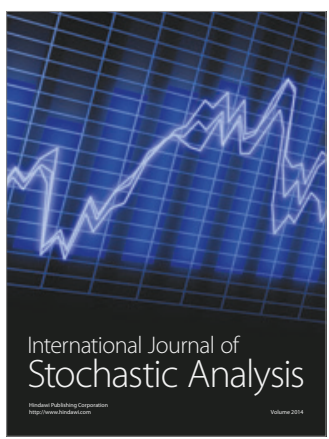

Probability and Statistics
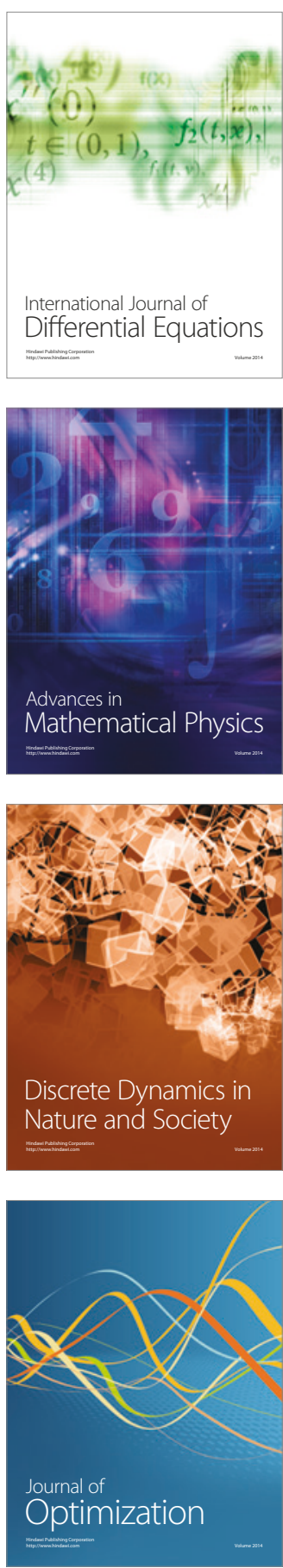